\title{
Quantitative Low-Dose Computed Tomography of the Lung Parenchyma and Airways for the Differentiation between Chronic Obstructive Pulmonary Disease and Asthma Patients
}

\author{
Huai Chen $^{\text {a,d }}$ Qing-si Zeng ${ }^{\mathrm{a}}$ Min Zhang $^{\mathrm{d}}$ Rong-chang Chen ${ }^{\mathrm{b}}$ Ting-ting Xia ${ }^{\mathrm{a}}$ \\ Wei Wang ${ }^{c}$ Xiaoyin $\mathrm{Xu}^{\mathrm{d}}$ Yubao Guan ${ }^{\mathrm{a}}$ \\ ${ }^{a}$ Department of Radiology and ${ }^{b}$ National Key Laboratory of Respiratory Diseases, the First Affiliated Hospital \\ of Guangzhou Medical University, Guangzhou, China; Departments of ${ }^{\mathrm{C}}$ Medicine and ${ }^{\mathrm{d}}$ Radiology, Brigham and \\ Women's Hospital, Harvard Medical School, Boston, MA, USA
}

\section{Keywords}

Chronic obstructive pulmonary disease - Asthma .

Computed tomography $\cdot$ Pulmonary function test .

Quantitative measurements

\begin{abstract}
Background: It is difficult to differentiate between chronic obstructive pulmonary disease (COPD) and asthma in clinics; therefore, for diagnostic purposes, imaging-based measurements could be beneficial to differentiate between the two diseases. Objectives: We aim to analyze quantitative measurements of the lung and bronchial parameters that are provided by low-dose computed tomography (CT) to differentiate COPD and asthma from an imaging perspective. $\mathbf{M a -}$ terials and Methods: 69 COPD patients, 52 asthma patients, and 20 healthy subjects were recruited to participate in CT imaging and pulmonary function tests (PFTs). Comparative analysis was performed to identify differences between COPD and asthma in CT measurements. PFT measurements enabled validation of the differentiation between COPD and asthma patients. Results: There were significant differences among the COPD, asthma, and healthy control groups. The differences were more significant among the following: inspiratory emphysema index (EI)_950 (\%), expiratory lung vol-
\end{abstract}

\section{KARGER}

(C) 2017 S. Karger AG, Basel

E-Mail karger@karger.com

www.karger.com/res ume, expiratory mean lung density (MLD), and expiratory $\mathrm{EI}_{-950}(\%)$ and $\mathrm{EI}_{-850}(\%)$. The COPD group had a significantly higher $\mathrm{EI}_{-950}(\%)$ than the asthma group ( $\left.p=0.008\right)$. There were significant differences among the three groups in lumen area (LA), wall area (WA), total area, and Pi10WA. The asthma group had significantly higher WA\%/WV\% than both the COPD $(p=0.002)$ and the control group $(p=0.012)$. There was high sensitivity in $\mathrm{El}_{-950}(\%), \mathrm{El}_{-850}(\%)$ and expiratory MLD in the parenchyma and high sensitivity in LA and Pi10WA in small airways in the differential diagnosis of COPD and asthma. Conclusion: To aid the diagnosis, CT can provide quantitative measurements to differentiate between COPD and asthma patients.

(c) 2017 S. Karger AG, Basel

\section{Introduction}

Accurate diagnosis of pulmonary diseases is of great significance to treat and manage patients well. Although pulmonary function tests (PFTs) are generally accepted as the common standard for diagnosing many pulmonary

Yubao Guan

Department of Radiology

The First Affiliated Hospital of Guangzhou Medical University Guangzhou, Guangdong 510120 (China) E-Mail yubaoguan69@163.com

Xiaoyin Xu

Department of Radiology, Brigham and Women's Hospital, Harvard Medical School Boston, MA 02115 (USA)

E-Mail xxu@bwh.harvard.edu 
Table 1. Baseline and demographic characteristics of the healthy control, COPD, and asthma patients

\begin{tabular}{lclc}
\hline & Control $(n=20)$ & COPD $(n=69)$ & Asthma $(n=52)$ \\
\hline Age, years & $42.8 \pm 12.0(23-67)$ & $46.6 \pm 12.59(29-78)$ & $46.6 \pm 12.6(18-65)$ \\
Male/female & $10 / 10$ & $68 / 1$ & $25 / 27$ \\
BMI & $23.58 \pm 3.93$ & $21.6 \pm 3.40$ & $23.33 \pm 3.70$ \\
BSA, $\mathrm{m}^{2}$ & $1.68 \pm 0.19$ & $1.64 \pm 0.21$ & $1.67 \pm 0.21$ \\
\hline
\end{tabular}

BSA $=0.20247 \times$ height $^{0.725} \times$ weight $^{0.425}$, where height was measured in meters and weight was measured in kilograms. diseases, the tests have a substantial rate of false positives because lungs have a strong self-compensating capability that may mask signs of diseases at early stages [1]. In addition, PFTs require the cooperation of patients for an accurate reading, which is not always achievable with pediatric patients or patients with advanced stages of pulmonary diseases. A radiological examination may provide an independent approach for assessing lung diseases or can be used in combination with PFTs for accurate and early diagnosis $[2,3]$. In a study comparing chronic obstructive pulmonary disease (COPD) patients and asthma patients, we evaluated how low-dose computed tomography (CT) and its quantitative analysis may be used to diagnose and differentiate between COPD and asthma.

COPD and asthma [4] are two prevalent pulmonary diseases, whose diagnosis and, in particular, differentiation remain challenging in clinics due to the overlap in symptoms between the two diseases. PFTs alone cannot differentiate to diagnose the right condition $[5,6]$. CT, however, can provide imaging-based characteristics about the lungs including measurements on lung parenchyma for detailed analyses of individual bronchi, such as small airways. Despite this promise, there lacks a way to comprehensively evaluate the CT measurements so as, again, to differentiate between the two diseases. In recent years, it has been recognized that a small airway, which is typically defined as that which has an internal diameter of less than $2 \mathrm{~mm}$, may represent a potential focus for treating COPD and asthma [7, 8]. Specifically, CT can provide valuable information about these areas in such a way that PFTs, including lung volume (LV), mean lung density (MLD), emphysema index (EI) ${ }_{-910}(\%), \mathrm{EI}_{-950}$ (\%), $\mathrm{EI}_{-850 /-950}(\%)$, and other parameters in both inspiratory and expiratory phases [9-12], cannot. We postulate that CT can be explored to find the parameters that can differentiate between COPD and asthma [11, 13-16]. As such, we hypothesized that quantitative features of CT can differentiate between COPD and asthma so as to allow an accurate diagnosis of each disorder. Thus, the ob- jective of this study was to identify the CT measurements that can best differentiate between COPD and asthma.

\section{Materials and Methods}

\section{Population and Ethics}

The study, which was approved by the institutional review board on research ethics, was conducted between January 2014 and June 2015 in Guangzhou, China. All the subjects were informed of the objective of the study and gave consent to participate. We recruited 20 healthy nonsmokers as the control group within which the subjects had no history of contacting dusts and any other known respiratory diseases. The subjects were recruited under the condition that they had had no CT scan in the past year and would not take part in CT-related research within the coming year so as to reduce their exposure to radiation. We recruited 69 COPD patients. The number of subjects of the comparison group in each COPD stage, as defined by the 2013 Global Initiative for Chronic Obstructive Lung Disease (GOLD) standard [5], included $24 / 69(35 \%)$ in stage I, $14 / 69$ (20\%) in stage II, $15 / 69$ (22\%) in stage III, and 16/69 (23\%) in stage IV. We recruited 52 asthma patients. The final diagnosis of all the COPD and asthma patients was confirmed after clinical examinations by physicians who followed universally accepted criteria. COPD and asthma of all patients were in the stable phase. The baseline and demographic characteristics of the study subjects are provided in Table 1.

\section{Computed Tomography}

A 16-detector row Toshiba Aquilion CT scanner was used to image all the subjects. The scanner was calibrated each day before scanning. The scanning parameters were tube voltage $120 \mathrm{kV}$, tube current $60 \mathrm{~mA}$, helical pitch 0.938 , rotation time $0.5 \mathrm{~s}$, scanned slice thickness $1.0 \mathrm{~mm}$, and interval $1.0 \mathrm{~mm}$. Reconstructed image size was $512 \times 512$ pixels. The volume dose index (CTDI vol) and the exposure length $(\mathrm{L})$ along the direction of the patient were recorded in the patients with CT scanning dose report. The following formula was used: $\mathrm{ED}=\mathrm{CTDI}$ vol $\times \mathrm{L} \times \mathrm{W}$ (where ED is the effective dose and $\mathrm{W}$ is the chest weight index of $0.017 \mathrm{mSv} /(\mathrm{mGy} \times$ $\mathrm{cm})$ ). Our results showed that the dose-length product range was 82.4-98.4 $\mathrm{mGy} \times \mathrm{cm}$ with an average of $89.5 \pm 5.3 \mathrm{mGy} \times \mathrm{cm}$. The effective radiation dose range was $1.40-1.69 \mathrm{mSv}$ with an average of $1.51 \pm 0.09 \mathrm{mSv}$. This dosage was lower than $10 \mathrm{mSv} / \mathrm{year}$ as required by the International Commission on Radiological Protection (ICRP).
Differentiation between COPD and Asthma Patients
Respiration 2017;94:366-374 DOI: $10.1159 / 000478531$ 
All the subjects were briefly trained to take and hold deep breaths and then to exhale completely. It was determined that all the subjects were able to perform the above breathing regimen satisfactorily prior to their inclusion in the experiments. During the CT examination, we visually assessed the breath of the subjects to identify their inspiratory and expiratory phases for accurate CT measurements. Also, during the CT examination, all subjects were found to have no conditions other than COPD or asthma for the two comparison groups. Two scans were performed at the inspiratory and expiratory phases for each subject.

All the images were analyzed using the commercial software LungCAD_SVM2.0.1 (Neusoft Medical Systems, Shenyang, China). We also used the software to automatically segment the lungs from the chest wall, mediastinum, large blood vessels, and bronchi. We then used the software to measure LV, MLD, EI-910 (\%), EI-950 (\%), $\mathrm{EI}_{-850}$ (\%), and the ratio of $\mathrm{EI}_{-850}$ to $\mathrm{EI}_{-950}\left(\mathrm{EI}_{-850 /-950}\right)(\%)$ in the inspiratory phase, MLD expiratory over inspiratory ratio (MLD E/I), the EI change of percent voxels between -850 and -950 $\mathrm{HU}$ on paired expiratory and inspiratory scans (EI-850/-950 expiratory-inspiratory E-I) (\%), and the percentage change of $\mathrm{EI}_{-850}$ on paired expiratory and inspiratory scans (EI-850 E-I) (\%).

Next, we used the software VIDA Appollo Version 1.2 to conduct 3D quantitative measurements of the airways [17-20]. We used the software to reconstruct the tree structure of the airways from generation $0-7$ of the bronchi. At this step, we obtained the cross-sectional area of the bronchi. We selected one apical and two basal segmental airways, the apical segmental bronchus of the right upper lobe (RB1), the posterior basal segmental bronchus of the right lower lobe (RB10), and the posterior basal segmental bronchus of the left lower lobe (LB10) as the target bronchial tubes for detailed analysis. We measured their lumen area (LA) and wall area (WA), and L of the 3rd-generation bronchi; wall volume $(\mathrm{WV})$ is WA $\times \mathrm{L}$. We calculated LV of each bronchus as area multiplied by length. We applied the body surface area (BSA) method to adjust the calculation to reduce the effect of each subject's height and weight on the above measurements. We measured the lumen perimeter $(\mathrm{Pi})$ on three different segments of bronchi and calculated the average of the three measurements. Pi10 (square root of the airway wall area at $\mathrm{Pi}$ of $10 \mathrm{~mm}$ ) is defined as the airways with a Pi of $10 \mathrm{~mm}$.

\section{Pulmonary Function Tests}

All the subjects underwent PFTs at 1 week after the CT examination. We used Cosmed Quark PFT (Cosmed, Rome, Italy) and Sensor Medics Vmax 229 (Sensor Medics Co., Yorba Linda, CA, USA) to measure the pulmonary functions of the subjects. PFTs were performed on all the subjects before and after bronchodilator (salbutamol $400 \mu \mathrm{g}$ ) administration via a spacer. We measured the total lung capacity (TLC), residual volume (RV), RV/TLC (\%), forced expiratory volume in the first second/forced vital capacity (FEV1/FVC) (\%), FEV1 (\%pred), FVC (\%pred), peak expiratory flow (\%pred), maximal mid-expiratory flow (\%pred), forced expiratory flow $(\mathrm{FEF})_{25 \%}$ (\%pred), $\mathrm{FEF}_{50 \%}$ (\%pred), post-bronchodilator FEV1 (\%pred), and post-bronchodilator FEV1/FVC (\%).

\section{Statistical Analysis}

All quantitative analyses are presented as mean \pm standard deviation. In comparing groups, if the data were determined to have a normal distribution with equal variations, analysis of variance was used. Otherwise, we used the Kruskal-Wallis $H$ test with Bon-
Table 2. Comparison of PFT measurements for asthma patients and COPD patients

\begin{tabular}{lrcc}
\hline & Asthma $(n=52)$ & COPD $(n=69)$ & $p$ value \\
\hline FEV1, \%pred & $61.67 \pm 22.80$ & $61.52 \pm 27.63$ & 0.99 \\
FVC, \%pred & $90.14 \pm 14.02$ & $89.34 \pm 22.06$ & 0.11 \\
FEV1/FVC, \% & $56.02 \pm 16.42$ & $51.01 \pm 14.64$ & 0.001 \\
FEF $_{25 \%}$ \% \%pred & $27.75 \pm 30.35$ & $26.31 \pm 14.71$ & 0.18 \\
FEF $_{50 \%}$ \%pred & $31.68 \pm 27.08$ & $26.76 \pm 18.33$ & 0.012 \\
MMEF, \%pred & $28.15 \pm 26.31$ & $25.47 \pm 15.82$ & 0.06 \\
PEF, \%pred & $59.45 \pm 26.22$ & $64.38 \pm 29.86$ & 0.37 \\
RV, \%pred & $148.99 \pm 49.22$ & $152.49 \pm 47.78$ & 0.72 \\
TLC, \%pred & $105.54 \pm 14.24$ & $110.33 \pm 14.83$ & 0.11 \\
RV/TLC, \%pred & $91.57 \pm 54.17$ & $135.74 \pm 32.11$ & $<0.001$ \\
\hline
\end{tabular}
flow.

MMEF, maximal mid-expiratory flow; PEF, peak expiratory

ferroni correction. For data subject to frequency distribution anal$y$ sis, if the number of theoretical frequency $T<5$ was less than onefifth of the total number of classes, the $\chi^{2}$ test was used. When the number of $\mathrm{T}<5$ was greater than one-fifth of the total number of classes, the Fisher exact test was used. SPSS 22.0 and GraphPad Prism 6.01 were used for all analyses. $p<0.05$ indicated statistical significance.

\section{Results}

\section{Differences in PFT among COPD and Asthma \\ Patients}

Quantitative PFT data were collected from 52 asthma and 69 COPD patients (Table 2). Statistical analysis shows that asthma patients had significantly higher FEV1/FVC (\%) and $\mathrm{FEF}_{50 \%}(\%)$ than the COPD patients $(p<0.05)$. However, asthma patients had significantly lower RV/ TLC $(\%)$ than COPD patients $(p<0.001)$. For any other parameters, there were no statistically significant differences.

\section{CT Differences in Lung Parenchyma among Healthy Control, COPD, and Asthma Patients}

The quantitative analyses of CT results from the lung parenchyma for the groups of healthy subjects, COPD, and asthma patients are shown in Table 3. Statistically significant differences of four parameters were observed among the three groups: EI $_{-950}$ (\%) of the inspiratory phase, LV and MLD of the expiratory phase, and the expiratory $\mathrm{EI}_{-850}(\%)$ in air trapping measurement. The COPD group had significantly higher readings than the 
Table 3. Comparison of quantitative CT of lung parenchyma among the healthy control, COPD patients, and asthma patients

\begin{tabular}{lcccc}
\hline & Asthma $(n=52)$ & COPD $(n=69)$ & Control $(n=20)$ & $p$ value \\
\hline Inspiratory phase & & & & \\
LV & $4.85 \pm 1.01$ & $5.65 \pm 0.88$ & $4.75 \pm 1.23$ & $<0.001^{\#, \&}$ \\
MLD, Hu & $-858.45 \pm 42.72$ & $-901.96 \pm 28.57$ & $-843.32 \pm 31.85$ & $<0.001^{\#, ~ \& ~}$ \\
EI_-850, \% & $77.50 \pm 9.34$ & $69.90 \pm 13.95$ & $65.46 \pm 15.74$ & $<0.001^{\#, ~ \& ~}$ \\
EI_950, \% & $10.67 \pm 16.63$ & $43.84 \pm 12.64$ & $1.84 \pm 1.90$ & $<0.001^{*}, \#, \&$ \\
Expiratory phase & & & & \\
LV & $2.79 \pm 0.81$ & $3.25 \pm 0.74$ & $2.07 \pm 0.68$ & $<0.001^{*}, \#, \&$ \\
MLD, Hu & $-750.22 \pm 57.09$ & $-811.91 \pm 50.00$ & $-675.88 \pm 60.05$ & $<0.001^{*}, \#, \&$ \\
EI_950, \% & $2.57 \pm 8.31$ & $22.48 \pm 12.53$ & $0.08 \pm 0.19$ & $<0.001^{\#, \&}$ \\
Air trapping & & & & \\
Expiratory EI_850, \% & $24.22 \pm 20.64$ & $51.91 \pm 14.51$ & $7.92 \pm 13.31$ & $<0.001^{*}, \#, \&$ \\
MLD E/I & $0.88 \pm 0.06$ & $0.90 \pm 0.04$ & $0.80 \pm 0.08$ & $<0.001^{*}, \#$ \\
\hline
\end{tabular}

${ }^{*} p<0.05$ indicates SD between the control and asthma groups. ${ }^{*} p<0.05$ indicates SD between the control and COPD groups. ${ }^{\circledR} p<0.05$ indicates SD between the COPD and asthma groups.

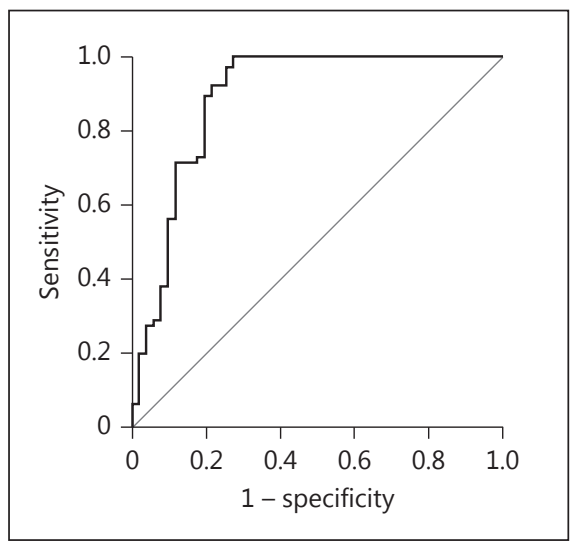

Fig. 1. ROC curve shows the performance of using EI-950 (\%) in the inspiratory phase to differentiate between COPD and asthma. AUC was 0.889 and cutoff was $6.05 \%$.

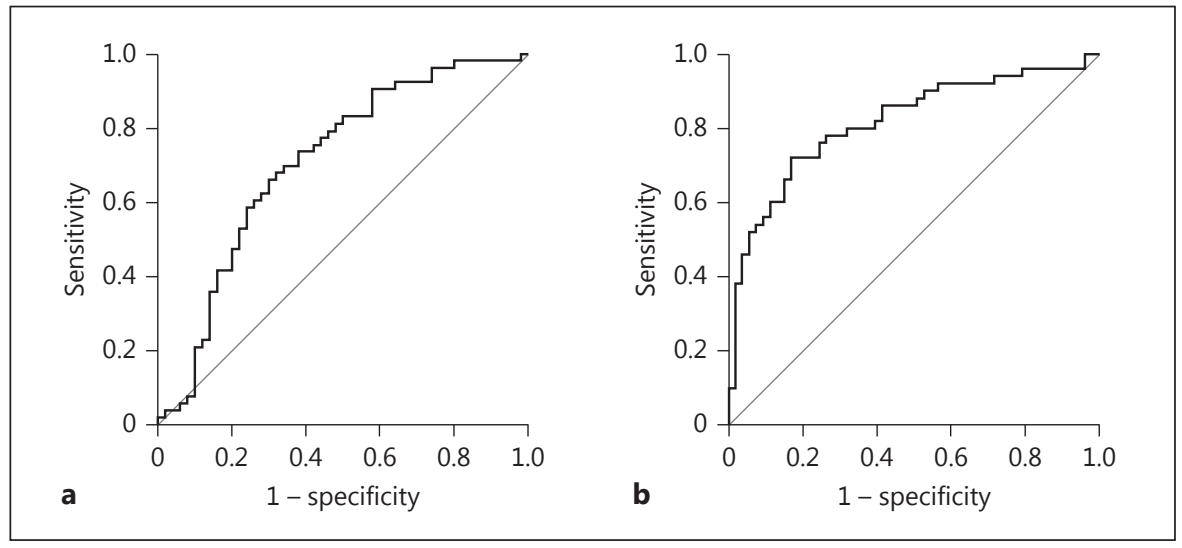

Fig. 2. a ROC curve of LV in the expiratory phase to differentiate between COPD and asthma. AUC was 0.708 and cutoff was 2.89 L. b ROC curve of MLD in the expiratory phase to differentiate between COPD and asthma. AUC was 0.814 and cutoff was -795.60 . asthma group, which, in turn, were significantly higher than the control group. Pairwise comparison shows that in the inspiratory phase the COPD group had higher LV, $\mathrm{MLD}$, and $\mathrm{EI}_{-850}$ (\%) than the asthma and control groups, though there was no statistically significant difference between the asthma and control groups. The COPD group had a significantly higher EI-950 (\%) than the asthma group ( $p=0.008$ ); both the COPD and asthma groups had significantly higher $\mathrm{EI}_{-950}(\%)$ than the control group $(p<0.001$, COPD; $p=0.02$, asthma). The resulting receiver operating characteristic (ROC) curve of $\mathrm{EI}_{-950}(\%)$

Differentiation between COPD and Asthma Patients of the inspiratory phase is shown in Figure 1. The area under the curve (AUC) was 0.889 .

In the expiratory phase, the COPD group had significantly larger LV than the asthma group $(p=0.008)$. Both the COPD and asthma groups had significantly larger LV than the control group $(p<0.001$, COPD; $p=0.001$, asth$\mathrm{ma})$. The COPD group had significantly lower MLD than the asthma group $(p<0.001)$ and both the COPD and asthma groups had significantly lower MLDs than the control group $(p<0.001)$. Figure 2 plots the ROC curves of using LV and MLD that differentiates between the 
Table 4. Comparison of CT measurements of proximal airways in inspiratory phase among the healthy control, COPD patients, and asthma patients

\begin{tabular}{|c|c|c|c|c|}
\hline & Asthma $(n=52)$ & $\operatorname{COPD}(n=69)$ & Control $(n=20)$ & $p$ value \\
\hline $\mathrm{WA} / \mathrm{BSA}, \mathrm{mm}^{2} / \mathrm{m}^{2}$ & $18.65 \pm 3.39$ & $21.19 \pm 4.50$ & $20.03 \pm 3.39$ & $0.001^{\#}$ \\
\hline $\mathrm{LA} / \mathrm{BSA}, \mathrm{mm}^{2} / \mathrm{m}^{2}$ & $10.53 \pm 3.16$ & $13.15 \pm 3.91$ & $12.56 \pm 3.04$ & $<0.001^{*, \#}$ \\
\hline $\mathrm{TA} / \mathrm{BSA}, \mathrm{mm}^{2} / \mathrm{m}^{2}$ & $29.18 \pm 7.20$ & $34.33 \pm 8.18$ & $36.22 \pm 8.10$ & $<0.001^{*, \#}$ \\
\hline Length/BSA, $\mathrm{mm} / \mathrm{m}^{2}$ & $8.06 \pm 1.78$ & $7.91 \pm 2.04$ & $7.29 \pm 1.81$ & 0.25 \\
\hline $\mathrm{WV} / \mathrm{BSA}, \mathrm{mm}^{3} / \mathrm{m}^{2}$ & $237.73 \pm 64.03$ & $264.19 \pm 75.10$ & $238.73 \pm 59.86$ & $0.017^{\#}$ \\
\hline $\mathrm{LV} / \mathrm{BSA}, \mathrm{mm}^{3} / \mathrm{m}^{2}$ & $136.12 \pm 43.68$ & $161.77 \pm 50.07$ & $148.86 \pm 42.13$ & $0.002^{\#}$ \\
\hline $\mathrm{TV} / \mathrm{BSA}, \mathrm{mm}^{3} / \mathrm{m}^{2}$ & $373.84 \pm 102.77$ & $425.97 \pm 122.77$ & $387.59 \pm 99.94$ & $0.006^{\#}$ \\
\hline WA\%/WV\% & $64.29 \pm 3.61$ & $62.42 \pm 2.63$ & $62.00 \pm 2.99$ & $0.001^{*, \#}$ \\
\hline
\end{tabular}

${ }^{*} p<0.05$ indicates SD between the control and asthma groups. ${ }^{*} p<0.05$ indicates SD between the COPD and asthma groups.

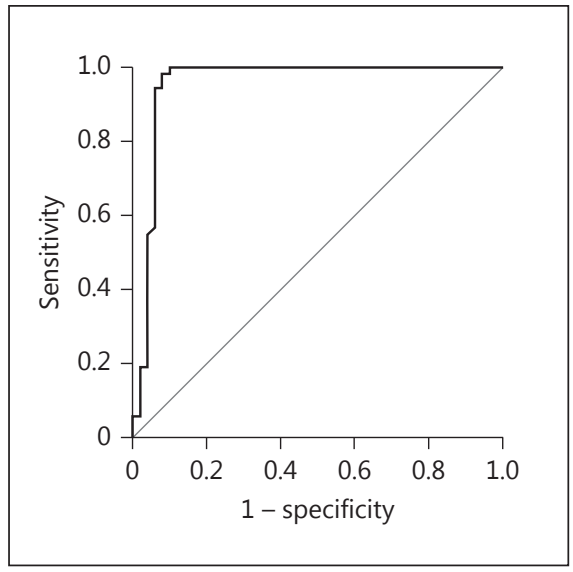

Fig. 3. ROC curve of $\mathrm{EI}_{-950}(\%)$ in the expiratory phase. AUC was 0.955. Cutoff was $5.77 \%$.

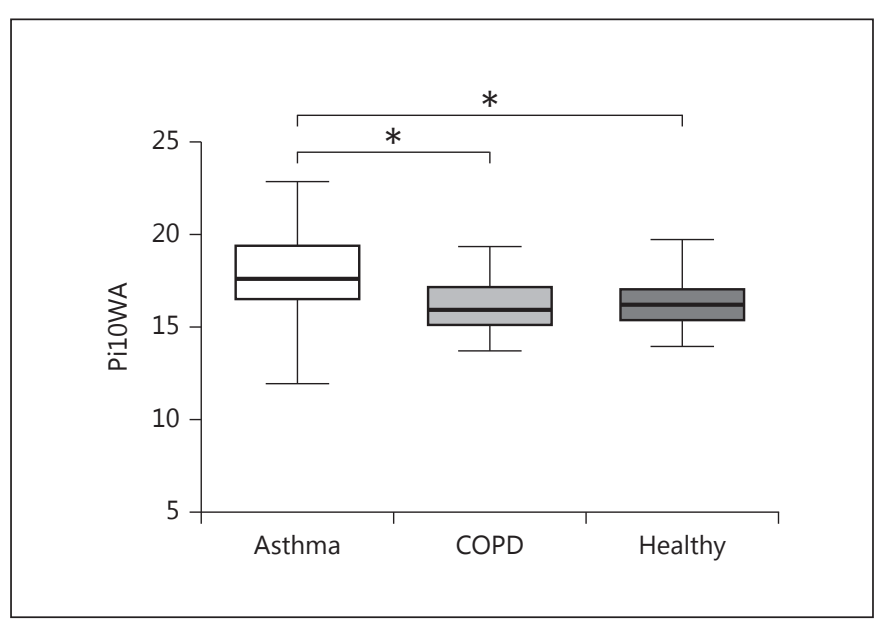

Fig. 4. Pairwise comparisons between the COPD, asthma, and the control groups in Pi10WA.
COPD and the asthma groups. The AUCs were 0.708 and 0.814 , respectively. The highest differentiation power was obtained when using EI-950 (\%) in the expiratory phase to separate the COPD and asthma groups. The ROC curve of $\mathrm{EI}_{-950}(\%)$ to differentiate between the COPD and asthma groups was shown in Figure 3 and had an AUC of 0.955.

In terms of air trapping, in the expiratory phase the COPD group had significantly higher $\mathrm{EI}_{-850}(\%)$ than the asthma group $(p<0.001)$. Both the COPD and asthma groups had significantly higher $\mathrm{EI}_{-850}(\%)$ than the control group.

\section{CT Differences in Proximal Airways among Healthy Subjects, COPD, and Asthma Patients}

We analyzed CT results in measuring proximal airways, RB1, RB10, and LB1, among the three groups (Table 4). There were statistically significant differences among the three groups in the RB1, RB10, and LB1 measurements. Pairwise comparison showed that the asthma group had higher WA/BSA than the COPD group $(p<$ $0.001)$. The asthma also had lower LA/BSA than the COPD $(p<0.001)$ and the control group $(p=0.037)$. Similarly, the asthma group had higher TA/BSA than the COPD $(p<0.001)$ and the control group $(p=0.001)$. In terms of WV/BSA, LV/BSA, and total volume/BSA, the asthma group had statistically significant differences relative to the COPD group ( $p$ values were $0.02,0.002$, and 0.006 , respectively). The asthma group had significantly higher WA\%/WV\% than both the COPD $(p=0.002)$ and the control group $(p=0.012)$. In terms of Pi10WA measurement, the result of the asthma group was $17.82 \pm 2.43$ $\mathrm{mm}^{2}$. The result of the COPD group was $16.10 \pm 1.28$ $\mathrm{mm}^{2}$ and that of the control group was $16.37 \pm 1.42 \mathrm{~mm}^{2}$. Statistically, the asthma group had significantly higher 

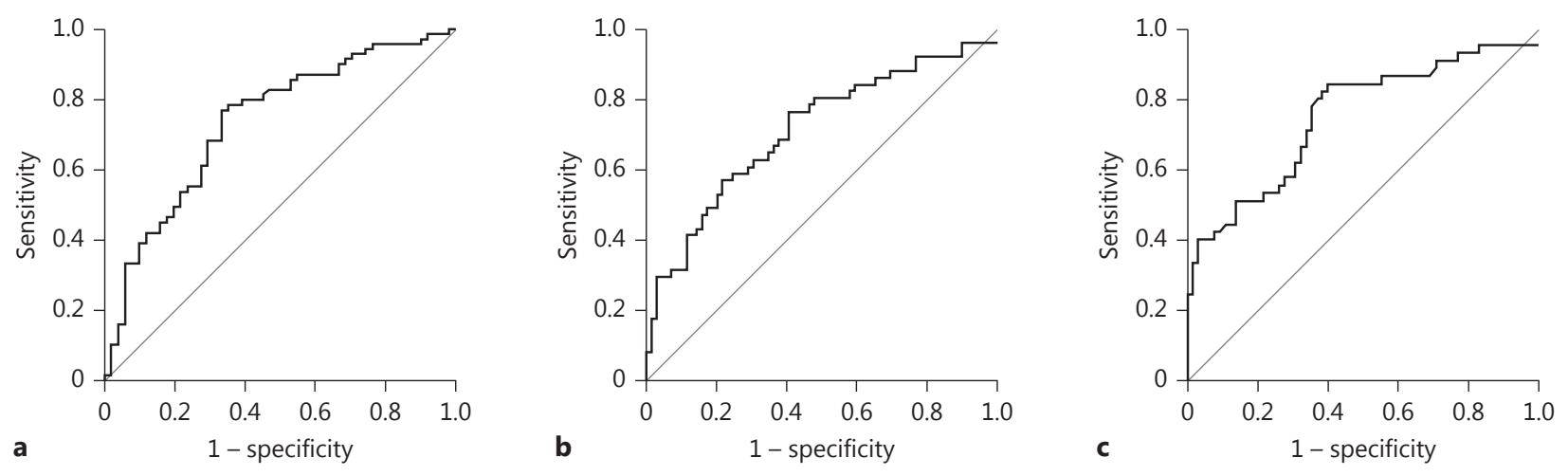

Fig. 5. ROC curves show the performance of using LA/BSA (a), WA\%/WV\% (b), and Pi10WA (c) in proximal airway measurements to differentiate between COPD and asthma. The AUCs were 0.736, 0.711, and 0.751, respectively. The cutoffs were $10.26 \mathrm{~mm}^{2} / \mathrm{m}^{2}, 63.02 \%$, and $16.33 \mathrm{~m}^{2}$, respectively.

Pi10WA than both the COPD $(p<0.001)$ and the control group $(p=0.008)$. The result of pairwise comparisons of the COPD, asthma, and the control groups in Pi10WA is shown in Figure 4, from which we can tell that there were statistically significant differences between any two groups in Pi10WA measurement. The ROC curves of LA/ BSA, WA\%/WV\%, and Pi10WA are plotted in Figure 5, in which the ROC curve of Pi10WA had, among the three, the highest AUC at 0.751 .

\section{Discussion}

Both COPD and asthma are disorders caused by small airways that have become restrained. Their diagnoses are usually based on the patient's symptoms, risk factors, and the presence of inflammation. However, differentiation between the two conditions remains challenging. Investigators have attempted to derive differentiating criteria based on causes, symptoms, saliva analysis, and pulmonary function testing. Nevertheless, still in practice, it is difficult for clinicians to tell the two conditions apart. In addition, studies have found that as high as $43 \%$ of COPD patients also had asthma and at least $46 \%$ of asthma patients also have been diagnosed with COPD [21].

In this study, we found that, in the inspiratory phase, COPD patients had significantly higher $\mathrm{EI}_{-950}$ (\%) than the control and asthma groups, indicating that COPD patients had more obvious emphysema changes than the asthma patients and, similarly, that asthma patients had

Differentiation between COPD and Asthma Patients more obvious emphysema changes than the control group. These findings led us to conclude that there was more damage to the lung tissues in the COPD patients than in the asthma patients that then was resulting in more severe emphysema. This result is consistent with previous studies that show that emphysema is a more prominent phenotype in COPD patients [22] but less so for asthmatic patients [23]. Our findings indicate that using inspiratory phase $\mathrm{EI}_{-950}$ (\%) as a criterion can lead to successful differentiation between COPD and asthma. Specifically, EI ${ }_{-950}(\%)$ had a sensitivity of $100 \%$, a specificity of $72.5 \%$, and AUC of 0.889 . In the expiratory phase measurement, $\mathrm{EI}_{-950}$ (\%), LV, and MLD may be further criteria to differentiate between COPD and asthma. For example, our analysis showed that the COPD group had significantly higher $\mathrm{EI}_{-950}$ (\%), LV, and MLD than both the asthma and the control groups. Furthermore, air trapping measurements revealed that, in the expiratory phase, the COPD patients had significantly higher $\mathrm{EI}_{-850}$ (\%) than the asthma group which, in turn, had significantly higher $\mathrm{EI}_{-850}$ (\%) than the control group. The COPD and asthma groups also had significantly higher MLD E/I than the control group, though there was no statistically significant difference between the two disease groups, indicating that MLD E/I by itself may not be suitable as a means to differentiate between COPD and asthma.

Previous CT studies have shown that there are differences in the characteristics of the airways between COPD and asthma patients [24-26]. Our quantitative analysis of CT on proximal airways showed that the asthma group

Respiration 2017;94:366-374 371 
had lower LA/BSA and TA/BSA but higher WA\%/WV\% and PI10WA than the control group, pointing to a remodeling of the proximal airways. However, there was no statistically significant difference in airway remodeling between the COPD and the control groups, indicating that there was no obvious airway remodeling in COPD. Similarly, we found that the asthma group had lower $\mathrm{WA}(\mathrm{WV}) / \mathrm{BSA}, \mathrm{LA}(\mathrm{LV}) / \mathrm{BSA}$, and TA(total volume)/ $\mathrm{BSA}$, but higher $\mathrm{WA} \% / \mathrm{WV} \%$ and Pi10WA than the COPD group, confirming findings by other investigators [27] that the airways of asthma patients tend to have smaller inner radii than those found in COPD patients [28-30]. Pathophysiological analysis confirmed that the airways of asthma patients have thicker basement membrane [31] and larger numbers of blood vessels [25] than COPD patients. Pairwise comparisons between the COPD and asthma groups in LA/BSA, WA\%/WV\%, and Pi10WA found that the AUCs of the three parameters are $0.736,0.711$, and 0.751 , respectively, meaning that the three parameters are approximately equal in the extent to which they differentiate between the two conditions.

When we used PFTs to differentiate between the COPD and asthma groups, we found that, on the one hand, the FEV1/FVC (\%) and $\mathrm{FEF}_{50 \%}$ (\%pred) of asthma patients were significantly larger than those of COPD patients, indicating that there was less restraining in the air flow in the asthma patients. The asthma group, on the other hand, had significantly lower RV/TLC (\%pred) than the COPD group, indicating that the COPD patients had greater air trapping than the asthma patients. Overall, these PFT results are consistent with those shown by CT. When comparing across the AUCs given by the three PFT parameters (FEV1/FVC (\%), $\mathrm{FEF}_{50 \%}$ (\%pred), RV/RLC (\%pred)) of PFT and the three CT parameters (LA/BSA, WA\%/WV\%, Pi10WA), RV/TLC (\%pred) had the largest AUC of 0.760 while the other two PFT parameters had lower AUCs. Hence, the AUCs of the CT parameters were more consistent and, overall, were higher than the AUCs of the PFTs, pointing to high efficacy when using CT to differentiate between COPD and asthma.

In our study, we observed that, in terms of baseline measurement, COPD tends to occur in males at an old age as there were 68 males and 1 female in the COPD patient cohort when recruitment was done for this study. This occurrence may be likely due to the fact that there are more male smokers than female smokers in the local population that our institution usually serves in practice. The average age of the COPD group was 65 , which was older than the asthma group. In terms of BMI, the COPD group had a smaller BMI than both the asthma and con- trol groups, which was an observation that is consistent with other investigators' findings [32]. Our deduction is that, on the one hand, as a chronic condition, COPD tends to affect older people. On the other hand, however, at stages I and II, COPD patients do not experience obvious deterioration of lung functions and, therefore, do not tend to seek medical intervention. When COPD patients start to have symptoms later on, they are often at an older age. In comparison, asthma tends to occur in people across a large range of ages and these patients generally experience clear symptoms and then tend to seek medical intervention promptly, making this group younger on average than the COPD group. The difference between the COPD and the asthma groups in BMI evaluation is likely due to the fact that air trapping in asthma patients is reversible, so when medication is administered promptly, patients do not undergo prolonged periods of oxygen shortage and are subsequently less affected physically by the disease. Larger experiments are necessary to examine the underlying demographic factors that affect the onset of COPD and asthma.

The contribution of this study is the demonstration that $\mathrm{CT}$, through its quantification results, can provide a rich set of parameters for differentiating between COPD and asthma. The parameters are based on both lung parenchyma and airways for different measurements, and, therefore, can be further investigated so as to design robust set criteria for an accurate differential diagnosis of COPD and asthma.

A potential limitation of our study is that it is primarily based on the quantification of CT images of subjects who had been diagnosed with COPD or asthma but not inflammation. In practice, CT images may not be specific in determining whether changes in airways are due to remodeling caused by asthma or inflammation [33]. In order to enable the deriving of a robust interpretation of CT images in such cases, medical and family history and current treatment [34] as well as reversibility by bronchodilators [35] should be included in the diagnostic process. When we combine quantitative measurements with qualitative evaluations of the lungs by high-resolution CT, we can expect to achieve higher accuracy not only in differentiating between COPD and asthma [36] but also in classifying their respective subtypes [37]. In the current study, we did not categorize COPD and asthma patients into their respective subtypes; however, with a large data set, it will be highly beneficial to make more detailed comparisons between the two diseases based on their CT biomarkers to guide clinical practice. In this study, we treated all the COPD patients as one cohort and all the asthma
372

Respiration 2017;94:366-374

DOI: $10.1159 / 000478531$
Chen/Zeng/Zhang/Chen/Xia/Wang/Xu/ Guan 
patients as another cohort for comparison to identify the most common CT biomarkers to differentiate between the two diseases. However, it is beneficial to further investigate how specific types of COPD and asthma patients may compare against each other from an imaging perspective. We will expand our studies in the future to investigate this problem.

In summary, this study demonstrates that $\mathrm{CT}$ can be a valuable technique to aid the accurate diagnosis of and differentiation between COPD and asthma. When combined with PFTs, we can expect an even higher sensitivity in differentiating between the two diseases. Quantitative modeling and analysis of CT scans of the lungs are quickly advancing our applications of CT to monitor the course of lung diseases and assess their prognoses [3, 20, 38]. Computer-aided analysis can also be used to reach quick and objective results in practice $[18,39,40]$. The accurate and prompt diagnosis of COPD and asthma can allow clinicians to better treat and manage the large populations of patients with these diseases.

\section{Acknowledgments}

The work of H. Chen was supported by the Science and Technology Planning Project of Guangdong Province, China (2014A020212608). The work of Q. Zeng was supported by Public Science and Technology Research Funds Projects of NHFPC of the P.R. China (201402013). The work of T. Xia was supported by the DepartmentofEducation ofGuangdong Province(2013KJCX0150) and Guangzhou Science Technology and Innovation commission (201300000190). The work of Y. Guan was supported by the National Nature Science Foundation of China (81371633) and Science and Technology Planning Project of Guangdong Province, China (2013B021800310). The work of X. Xu was supported by the National Institutes of Health (R01LM011415 and R01LM012434).

\section{References}

1 Pellegrino R, Viegi G, Brusasco V, Crapo RO, Burgos F, Casaburi R, Coates A, van der Grinten CP, Gustafsson P, Hankinson J, Jensen R, Johnson DC, MacIntyre N, McKay R, Miller MR, Navajas D, Pedersen OF, Wanger $\mathrm{J}$ : Interpretative strategies for lung function tests. Eur Respir J 2005;26:948-968.

2 Chen H, Chen RC, Guan YB, Li W, Liu Q, Zeng QS: Correlation of pulmonary function indexes determined by low-dose MDCT with spirometric pulmonary function tests in patients with chronic obstructive pulmonary disease. AJR Am J Roentgenol 2014;202:711718 .

3 Matsuoka S, Kurihara Y, Yagihashi K, Nakajima Y: Quantitative thin-section CT analysis of the enlargement and coalescence of lowattenuation clusters in patients with emphysema. Respiration 2007;74:136-141.

4 Mannino DM, Buist AS: Global burden of COPD: risk factors, prevalence, and future trends. Lancet 2007;370:765-773.

5 Vestbo J, Hurd SS, Agusti AG, Jones PW, Vogelmeier C, Anzueto A, Barnes PJ, Fabbri LM, Martinez FJ, Nishimura M, Stockley RA, Sin $\mathrm{DD}$, Rodriguez-Roisin R: Global strategy for the diagnosis, management, and prevention of chronic obstructive pulmonary disease: GOLD executive summary. Am J Respir Crit Care Med 2013;187:347-365.

6 Heaney LG, McGarvey LP: Personalised medicine for asthma and chronic obstructive pulmonary disease. Respiration 2017;93:153161

7 Macklem PT: The physiology of small airways. Am J Respir Crit Care Med 1998; 157:S181-S183.
8 Hogg JC, Chu F, Utokaparch S, Woods R, Elliott WM, Buzatu L, Cherniack RM, Rogers RM, Sciurba FC, Coxson HO, Pare PD: The nature of small-airway obstruction in chronic obstructive pulmonary disease. N Engl J Med 2004;350:2645-2653.

9 Hackx M, Bankier AA, Gevenois PA: Chronic obstructive pulmonary disease: CT quantification of airways disease. Radiology 2012;265: 34-48.

10 Busacker A, Newell JD Jr, Keefe T, Hoffman EA, Granroth JC, Castro M, Fain S, Wenzel S: A multivariate analysis of risk factors for the air-trapping asthmatic phenotype as measured by quantitative CT analysis. Chest 2009; 135:48-56.

11 Montaudon M, Lederlin M, Reich S, Begueret $\mathrm{H}$, Tunon-de-Lara JM, Marthan R, Berger P, Laurent F: Bronchial measurements in patients with asthma: comparison of quantitative thin-section CT findings with those in healthy subjects and correlation with pathologic findings. Radiology 2009;253:844-853.

12 Lee JS, Lee SM, Seo JB, Lee SW, Huh JW, Oh YM, Lee SD: Clinical utility of computed tomographic lung volumes in patients with chronic obstructive pulmonary disease. Respiration 2014;87:196-203.

13 Mets OM, de Jong PA, van Ginneken B, Kruitwagen CL, Prokop M, Oudkerk M, Lammers JW, Zanen P: CT air trapping is independently associated with lung function reduction over time. PLoS One 2013;8:e61783.

14 Matsuoka S, Washko GR, Dransfield MT, Yamashiro T, San Jose Estepar R, Diaz A, Silverman EK, Patz S, Hatabu H: Quantitative CT measurement of cross-sectional area of small pulmonary vessel in COPD: correlations with emphysema and airflow limitation. Acad Radiol 2010;17:93-99.
15 Fuld MK, Grout RW, Guo J, Morgan JH, Hoffman EA: Systems for lung volume standardization during static and dynamic MDCT-based quantitative assessment of pulmonary structure and function. Acad Radiol 2012;19:930-940.

16 Gao Y, Zhai X, Li K, Zhang H, Wang Y, Lu Y, Pan Z, Zhang L, Huang K, Zhai R: Asthma COPD overlap syndrome on CT densitometry: a distinct phenotype from COPD. COPD 2016;13:471-476.

17 Gupta S, Hartley R, Khan UT, Singapuri A, Hargadon B, Monteiro W, Pavord ID, Sousa AR, Marshall RP, Subramanian D, Parr D, Entwisle JJ, Siddiqui S, Raj V, Brightling CE: Quantitative computed tomography-derived clusters: redefining airway remodeling in asthmatic patients. J Allergy Clin Immunol 2014;133:729-738.e18.

18 Hartley RA, Barker BL, Newby C, Pakkal M, Baldi S, Kajekar R, Kay R, Laurencin M, Marshall RP, Sousa AR, Parmar H, Siddiqui S, Gupta S, Brightling CE: Relationship between lung function and quantitative computed tomographic parameters of airway remodeling, air trapping, and emphysema in patients with asthma and chronic obstructive pulmonary disease: a single-center study. J Allergy Clin Immunol 2016;137:1413-1422.e12.

19 Van Tho N, Wada H, Ogawa E, Nakano Y: Recent findings in chronic obstructive pulmonary disease by using quantitative computed tomography. Respir Investig 2012;50: 78-87.

20 de Oliveira HG, de Oliveira SM, Rambo RR, de Macedo Neto AV: Fissure integrity and volume reduction in emphysema: a retrospective study. Respiration 2016;91:471-479.
Differentiation between COPD and Asthma Patients
Respiration 2017;94:366-374 DOI: $10.1159 / 000478531$ 
21 Weatherall M, Travers J, Shirtcliffe PM, Marsh SE, Williams MV, Nowitz MR, Aldington S, Beasley R: Distinct clinical phenotypes of airways disease defined by cluster analysis. Eur Respir J 2009;34:812-818.

22 McDonough JE, Yuan R, Suzuki M, Seyednejad N, Elliott WM, Sanchez PG, Wright AC, Gefter WB, Litzky L, Coxson HO, Pare PD, Sin DD, Pierce RA, Woods JC, McWilliams AM, Mayo JR, Lam SC, Cooper JD, Hogg JC: Small-airway obstruction and emphysema in chronic obstructive pulmonary disease. N Engl J Med 2011;365:1567-1575.

23 Hong KY, Lee JH, Park SW, Joo JH, Kim DJ, Moon SH, Uh ST, Kim YH, Park CS, Park JS: Evaluation of emphysema in patients with asthma using high-resolution CT. Korean J Intern Med 2002;17:24-30.

24 Kosciuch J, Krenke R, Gorska K, Zukowska M, Maskey-Warzechowska M, Chazan R: Relationship between airway wall thickness assessed by high-resolution computed tomography and lung function in patients with asth$\mathrm{ma}$ and chronic obstructive pulmonary disease. J Physiol Pharmacol 2009;60(suppl 5):71-76.

25 Mohamed Hoesein FA, de Jong PA, Lammers JW, Mali WP, Mets OM, Schmidt M, de Koning HJ, Aalst C, Oudkerk M, Vliegenthart R, Ginneken B, van Rikxoort EM, Zanen P: Contribution of CT quantified emphysema, air trapping and airway wall thickness on pulmonary function in male smokers with and without COPD. COPD 2014;11:503-509.

26 Nakano Y, Muller NL, King GG, Niimi A, Kalloger SE, Mishima M, Pare PD: Quantitative assessment of airway remodeling using high-resolution CT. Chest 2002;122(6 suppl):271S-275S.
27 Oguma T, Hirai T, Fukui M, Tanabe N, Marumo S, Nakamura H, Ito H, Sato S, Niimi A, Ito I, Matsumoto H, Muro S, Mishima M: Longitudinal shape irregularity of airway lumen assessed by CT in patients with bronchial asth$\mathrm{ma}$ and COPD. Thorax 2015;70:719-724.

28 Kosciuch J, Krenke R, Gorska K, Zukowska M, Maskey-Warzechowska M, Chazan R: Airway dimensions in asthma and COPD in high resolution computed tomography: can we see the difference? Respir Care 2013;58: 1335-1342.

29 Shimizu K, Hasegawa M, Makita H, Nasuhara Y, Konno S, Nishimura M: Comparison of airway remodelling assessed by computed tomography in asthma and COPD. Respir Med 2011;105:1275-1283.

30 Kurashima K, Hoshi T, Takayanagi N, Takaku Y, Kagiyama N, Ohta C, Fujimura M, Sugita Y: Airway dimensions and pulmonary function in chronic obstructive pulmonary disease and bronchial asthma. Respirology 2012;17: 79-86.

31 Benfante A, Bellia M, Scichilone N, Cannizzaro F, Midiri M, Brown R, Bellia V: Airway distensibility by HRCT in asthmatics and COPD with comparable airway obstruction. COPD 2013; 10:560-566.

32 Shaya FT, Maneval MS, Gbarayor CM, Sohn K, Dalal AA, Du D, Scharf SM: Burden of COPD, asthma, and concomitant COPD and asthma among adults: racial disparities in a medicaid population. Chest 2009;136:405411.

33 Dournes G, Laurent F: Airway remodelling in asthma and COPD: findings, similarities, and differences using quantitative CT. Pulm Med 2012;2012:670414.
34 Patel BD, Coxson HO, Pillai SG, Agusti AG, Calverley PM, Donner CF, Make BJ, Muller NL, Rennard SI, Vestbo J, Wouters EF, Hiorns MP, Nakano Y, Camp PG, Nasute Fauerbach PV, Screaton NJ, Campbell EJ, Anderson WH, Pare PD, Levy RD, Lake SL, Silverman EK, Lomas DA, International COPD Genetics Network: Airway wall thickening and emphysema show independent familial aggregation in chronic obstructive pulmonary disease. Am J Respir Crit Care Med 2008;178:500505.

35 Fabbri LM, Romagnoli M, Corbetta L, Casoni G, Busljetic K, Turato G, Ligabue G, Ciaccia A, Saetta M, Papi A: Differences in airway inflammation in patients with fixed airflow obstruction due to asthma or chronic obstructive pulmonary disease. Am J Respir Crit Care Med 2003;167:418-424.

36 Gupta PP, Yadav R, Verma M, Agarwal D, Kumar M: Correlation between high-resolution computed tomography features and patients' characteristics in chronic obstructive pulmonary disease. Ann Thorac Med 2008;3: 87-93.

37 Gupta S, Siddiqui S, Haldar P, Entwisle JJ, Mawby D, Wardlaw AJ, Bradding P, Pavord ID, Green RH, Brightling CE: Quantitative analysis of high-resolution computed tomography scans in severe asthma subphenotypes. Thorax 2010;65:775-781.

38 Grydeland TB, Dirksen A, Coxson HO, Pillai SG, Sharma S, Eide GE, Gulsvik A, Bakke PS: Quantitative computed tomography: emphysema and airway wall thickness by sex, age and smoking. Eur Respir J 2009;34:858-865.

39 Burrowes KS, Doel T, Brightling C: Computational modeling of the obstructive lung diseases asthma and COPD. J Transl Med 2014; 12(suppl 2):S5.

40 Goldin JG: Quantitative CT of the lung. Radiol Clin North Am 2002;40:145-162. 